\title{
A Clinical Trial: Repeated Transcranial Direct Current Stimulation (tDCS) on Methamphetamine Craving: A Randomized, Sham-controlled Study
}

Mahsa Rohani Anaraki ${ }^{1}$ (D), Behrouz Dolatshahi ${ }^{1^{*}}$ (D), Masoud Nosratabadi ${ }^{1}$ (D), Mohsen Nouri Yalghouzaghaji ${ }^{1}$ (D), Sajede Rezaei Mashhadi ${ }^{1}$ (D)

\begin{tabular}{|c|c|}
\hline $\begin{array}{l}\text { Use your device to scan } \\
\text { and read the article online }\end{array}$ & Citation: Rohani Anaraki M, Dolatshahi B, Nosratabadi M, Nouri Yalghouzaghaji M, Rezaei Mashhadi S. Repeated Tran- \\
\hline 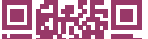 & scranial Direct Current Stimulation (tDCS) on Methamphetamine Craving; A Randomized, Sham-Controlled Study. Iranian \\
\hline Prys & Rehabilitation Journal. 2019; 17(4):385-394. http://dx.doi.org/10.32598/irj.17.4.385 \\
\hline 口ist. & dol ${ }^{z}$ http://dx.doi.org/10.32598/irj.17.4.385 \\
\hline
\end{tabular}

Article info:

Received: 09 Jul 2019

Accepted: 15 Oct 2019

Available Online: 01 Dec 2019

Keywords:

Transcranial Direct Current Stimulation, Methamphetamine craving, Dorsolateral prefrontal cortex

\section{ABSTRACT}

Objectives: The modulation of the dorsolateral prefrontal cortex (DLPFC) activity enhanced the prospects of substance use disorders rehabilitation, using non-invasive brain stimulation, such as transcranial Direct Current Stimulation (tDCS).

Methods: We carried out a randomized sham-controlled clinical trial to assess the effect of repeated tDCS at DLPFC on drug craving in 30 abstinent male methamphetamine users. The participants underwent 5 sessions of 20 minutes bilateral real or sham $2 \mathrm{mAtDCS}$ (anode right cathode left) of DLPFC. The Desire for Drug Questionnaire (DDQ) was used for assessing instant craving. The cue-induced craving was rated on a Visual Analog Scale (VAS) after the verbal induction of craving.

Results: The findings of this study indicated that cue-induced craving reduced significantly in tDCS related to sham $(\mathrm{P}<0.05)$, but $\mathrm{tDCS}$ did not significantly alter instant craving.

Discussion: The results indicated that repeated bilateral tDCS over DLPFC was not effective in reducing self-report instant craving, but reduced self-reported craving in the craving induction condition. 


\section{Highlights}

1- tDSC over dorsolateral prefrontal cortex reduces cue-induce craving in patients with methamphetamine use disorder.

2- tDCS over dorsolateral prefrontal cortex has no effect on instant craving in patients with methamphetamine use disorder.

\section{Plain Language Summary}

This clinical trial study performed to exam the effects of a neurotherapeutic intervention (tDSC) on drug craving in patients with methamphetamine use disorder. The findings indicate that this intervention is effective in reducing cue induced craving but has no effect on instant craving. this finding could be considered in prevention of relapsing in clinical intervention of drug use disorders and future investigations in this field.

\section{Introduction}

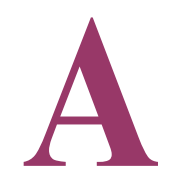

ll over the world, methamphetamine use is a serious threat to general health. According to the statistics released by the United Nations Office on Drugs and Crime in 2015, about 37 million people throughout the world were methamphetamine users. Amphetaminetype stimulants are the most common abused substances after cannabis and compared to other substance use disorders, amphetamine use disorder constitutes a considerable piece of the global weight of disease [1]. The number of methamphetamine addicts and demand for the treatment of methamphetamine-related conditions is increasing in many parts of the world, including southwest Asia [1]; however, no proper pharmacological treatment has yet been approved by the Food and Drug Administration [2], and behavioral interventions, as the main rehabilitation for these patients [3], have been successful only to some extent [4].

The difficulty of methamphetamine use disorder treatment is the high expectancy of relapse after abstinence [5]. Relapse may even occur after long periods of abstinence and is often accelerated during a craving or when drug-related cues are present $[5,6]$. Drug craving is one of the diagnostic criteria of substance use disorder according to the Diagnostic and Statistical Manual of Mental Disorders-Fifth Edition (DSM-5), and its reduction is an indicator of the success of addiction treatment or rehabilitation $[7,8]$.

Interconnected brain structures, such as the ventral tegmental area, amygdala, dorsomedial striatum, and
Prefrontal Cortex (PFC) are largely involved in the methamphetamine craving $[9,10]$. The different parts of the PFC have important roles in many cognitive processes, including control of inhibition and craving [11, 12]. Brain imaging studies show PFC impairment and its connection with response inhibition impairment in methamphetamine users [13]. Among the PFC regions, the Dorsolateral Prefrontal Cortex (DLPFC) is of special importance because of its involvement in motivation, reward, and decision-making; its connection with subcortical regions consists of stratum and cingulate cortex [8]. The DLPFC appears to assume a substantial job in the inhibition control through its connections with other brain regions [14]. In addition, the modulation of DLPFC activity using non-invasive brain stimulation has been found to have significant effects on the craving of patients with substance use disorder [15]. One of these methods is the transcranial Direct Current Stimulation (tDCS) that exerts a weak electrical current on the brain using electrodes; this changes the polarization of the nerve membrane, thereby changing its activity. "Anodal stimulation increases cortical excitability, while cathodal stimulation decreases it" [16].

The tDCS has emerged during the past several decades as a successful method for reducing psychiatric and neurological symptoms like depression, memory problems, and addiction [17]. For example, recent studies have shown the effectiveness of anodal stimulation of the right DLPFC in the reduction of craving for cocaine [18], nicotine [19], and alcohol [20]. However, only a few experimental studies have concentrated on the effect of tDCS on methamphetamine craving [21]. In one of these studies, one session of anodal stimulation 
of the right DLPFC immediately reduced drug craving in the rest condition but increased drug craving when participants were shown methamphetamine-related images. While one case study with a patient with methamphetamine use disorder has shown the effectiveness of repeated tDCS sessions with the same montage in controlling psychological stress and drug craving, preceding studies have shown that repeated stimulation can cause stronger and longer impact of tDCS in the clinical applications $[22,23]$.

We aimed in the present clinical trial at examining the effectiveness of repeated bilateral tDCS over DLPFC in those with methamphetamine use disorder.

\section{Methods}

Participants: A total of 36 patients attending rehab centers in Tehran were selected according to the inclusion and exclusion criteria, using a convenience sampling method; the participants were randomly assigned into active tDCS and sham tDCS groups. Of these 36 patients, 4 were excluded from the study because of drug use (see the exclusion criteria) and 2 other left the sessions without providing any reason; finally, 30 participants were incorporated in the analysis. The inclusion criteria were as follow: age 18 to 65 years old, male gender, methamphetamine use disorder based on the diagnostic criteria of DSM-5 for at least 12 months before starting the treatment, abstinence from any drug except cigarettes approved by multi-panel urine drug tests in the rehab centers for at least 1 week before starting the treatment, and at least $20 \%$ drug craving when faced with two images that induced craving before starting the stimulation sessions. The exclusion criteria were as follow: being under treatment for another psychiatric disorder (other than substance use disorders), being left-handed, having serious neurological disorder, taking any medication influencing the central nervous system, having the history of epilepsy, brain operation, tumors, intracranial implants, and clinically considerable head injury [21]. Participants continued their routine treatment, and no change was made into their treatment programs.

The present study was carried on according to the ethical principles of the Declaration of Helsinki and was approved by the Ethics Committee of the University of Social Welfare and Rehabilitation Sciences.

Study design: The present study is a random, doubleblind, experimental, sham-controlled research with pretest and posttest. Participants were randomly assigned to active tDCS and sham tDCS groups. The electric stimulation device was adjusted so that it provided active or sham stimulation. Neither the researcher who utilized the device to assess a patient's craving nor the patient knew if the stimulation was active or sham.

The sampling procedure had 4 stages, including the recruitment of participants, pretest, intervention, and posttest. These occurred in 5 separate individually-held sessions.

At the pretest, the demographic information of the participants was obtained, using the Basic Demographics and Substance Abuse Profile. Then, the participants completed the Positive and Negative Affect Schedule

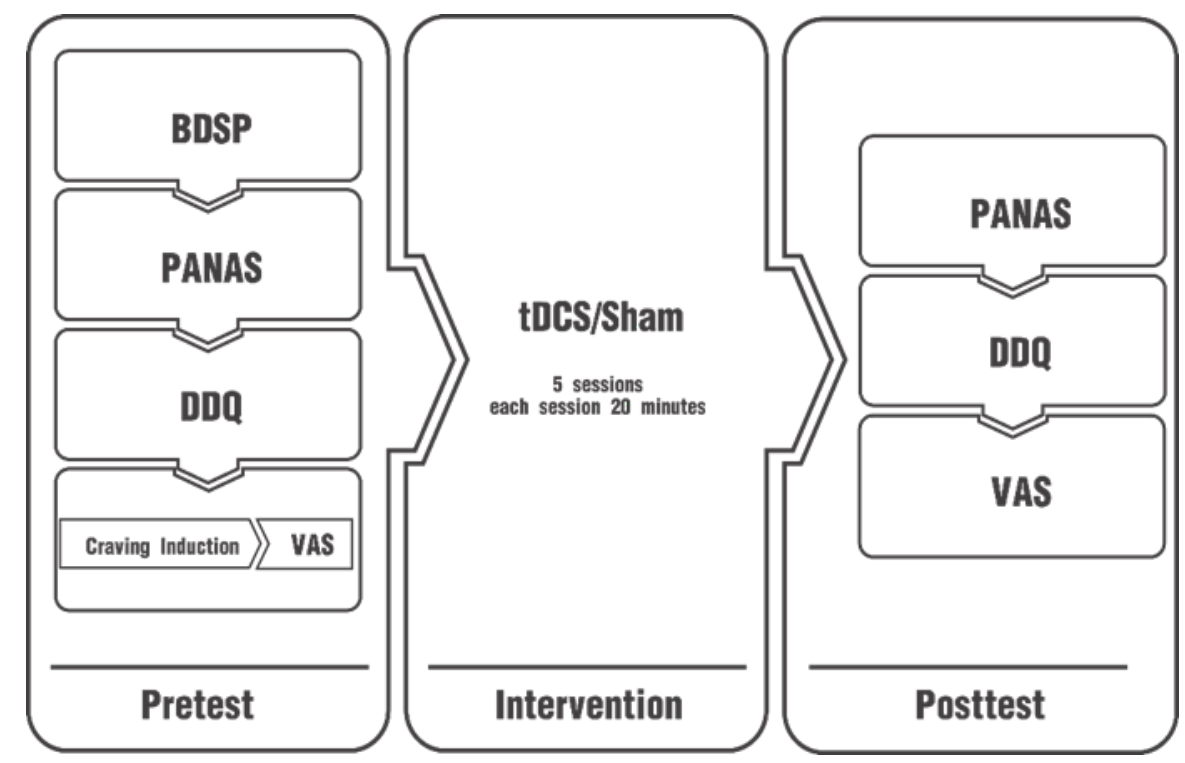

Figure 1. Intervention procedure 
(PANAS) and Desire for Drug Questionnaire (DDQ) and were put under craving induction for their craving level to be assessed; they showed their craving on a Visual Analog Scale (VAS).

Immediately after the pretest, the intervention was implemented. At the end of each session, the date of the next session was determined. The sessions were held at least 24 and at most 48 hours apart. In the final session, just at the end of stimulation, the posttest was administered. At the posttest, each patient was assessed, using the PANAS, the DDQ, the craving induction task, and the VAS, respectively (Figure 1).

DDQ: Is a self-report questionnaire developed by Franken et al., aimed at assessing instant craving for drugs [24]. It has 14 items rated on a 7-point Likert-type scale ranging from "thoroughly disagree" to "thoroughly agree". Using Cronbach's alpha method, Franken et al. reported the reliability of the total scale to be 0.85 [24].

The cue-induced craving: to assess induced craving, each patient was asked to describe 3 previous situations that had induced drug craving and led to drug use. They were asked to specifically describe the situation that had induced craving in them, and also their own emotional, physiological, and behavioral responses in that situation. At the same time, the researcher took notes from the patient's words. The situation and the recorded responses of the patient were, then, reviewed and the patients were asked to imagine the situation and to show their craving for methamphetamine on the VAS. In the present study, the VAS was a $100 \mathrm{~mm}$ line that ranged from 0 (I absolutely don't have a craving) to 100 (It is the strangest craving I have ever had). We, then, selected the situation that involved the highest level of craving for the patient and recorded the patient's response to that situation. This situation was used to induce craving at the posttest. Sinha et al. have used an almost similar method to induce carving for alcohol [25]. Because this could increase drug craving in the patients, they stayed in the treatment environment for 1 hour after the imagery process; then, their craving-related behaviors were examined by a skilled psychologist in order to make sure the patient was not intensely interested in drug use.

PANAS: This 20-item scale assesses two mood dimensions, i.e. positive and negative affect [26]. It was used in the present study to control participants' effect. Each subscale of the PANAS has 10 items and the items are rated on a 5-point Liker-type scale ranging from 1 (very low) to 5 (very high). Cronbach's alphas of 0.88 and 0.71 have been reported for the positive and negative affect scales, respectively.

Brain stimulation: a direct electric current was released, using a battery-driven simulator, and delivered to the brain by tow $5 \times 7 \mathrm{~cm}$ electrodes. In order to target the DLPFC, the Anode electrode was connected to the F4 region, and the cathode electrode was connected to the F3 region (that was $10 / 20$ based on the electroencephalography system). According to the previous studies showing the effectiveness of repeated tDCS, the electric current intensity was $2 \mathrm{~mA}$, and the stimulation duration was 20 minutes [17].

For the sham stimulation, electrodes with a similar montage were placed on the patient's head, but the simulator was slowly deactivated after 20 seconds. Therefore, the participants experienced itchiness at the start of stimulation but did not receive any stimulation in the rest of the session. This procedure helped in keeping subjects blind to the receptive stimulation condition. There were 5 sessions for each group.

Statistical analysis: the data analysis was performed, using SPSS v. 21. The 1-way Analysis of Covariance (ANCOVA) was used to compare the two groups in terms of induced craving and to remove the effect of the pretest. The differential t-test was used to compare the two groups in terms of instant craving and to remove the effect of the pretest. Before performing the analysis, we made sure that the participants were homogenized in terms of possible confounder variables. For this purpose, the Chi-square test was used to examine age, age at onset of methamphetamine use, duration of methamphetamine dependency, duration of drug abstinence, positive and negative affect, and pretest scores; the Chi-square test was used for the history of addiction to other drugs, and the Man Whitney U test was used to compare the two groups in terms of education and marital status.

\section{Results}

A total of 30 individuals were included in the present study. Table 1 presents the demographic characteristics of the participants. Table 2 presents information related to drug use. According to these tables, the active and sham tDCS groups are matched in terms of demographic and substance-related characteristics. In addition, at the pretest and posttest, the participants in the two groups were not significantly different in terms of positive and negative affect Table 2. 
Table 1. Demographic and substance-related characteristics of the participants in the sham and active tDCS groups

\begin{tabular}{|c|c|c|c|c|c|c|}
\hline Variables & Descriptions & All Subjects & Active & Sham & - & $\mathbf{P}$ \\
\hline \multirow{2}{*}{ Gender n (\%) } & Male & $30(100 \%)$ & $15(100)$ & $15(100)$ & \multirow{2}{*}{ - } & \multirow{2}{*}{-} \\
\hline & Female & 0 & 0 & 0 & & \\
\hline Age & Mean \pm SD & $\begin{array}{c}32.50 \\
9.27\end{array}$ & $\begin{array}{l}35.07 \\
11.57\end{array}$ & $\begin{array}{c}31.73 \\
6.29\end{array}$ & $\mathrm{t}=0.98$ & 0.33 \\
\hline \multirow{4}{*}{ Education n (\%) } & Guidance school & $9(30 \%)$ & $5(33.3)$ & $4(26.7)$ & \multirow{4}{*}{$U=103.5$} & \multirow{4}{*}{0.71} \\
\hline & High school & $12(40 \%)$ & $4(26.7)$ & $8(53.3)$ & & \\
\hline & Associate Degree & $7(23.3 \%)$ & $5(33.3)$ & $2(13.3)$ & & \\
\hline & Bachelor & $2(6.7 \%)$ & $1(6.7)$ & $1(6.7)$ & & \\
\hline \multirow{3}{*}{ Marital state } & Single & $15(50 \%)$ & $7(46.7)$ & $8(53.3)$ & \multirow{3}{*}{$\mathrm{U}=101$} & \multirow{3}{*}{0.65} \\
\hline & Married & $8(26.7 \%)$ & $5(33.3)$ & $3(20)$ & & \\
\hline & Divorced & $7(23.4 \%)$ & $3(20)$ & $4(26.7)$ & & \\
\hline \multirow{2}{*}{ Age at onset of methamphetamine use } & \multirow{6}{*}{ Mean \pm SD } & 26.20 & 28 & 24.40 & \multirow{2}{*}{$\mathrm{t}=1.21$} & \multirow{2}{*}{0.23} \\
\hline & & 8.18 & 9.16 & 6.92 & & \\
\hline \multirow[b]{2}{*}{ Days of methamphetamine abstinence } & & 43.26 & 31.40 & 55.13 & \multirow[b]{2}{*}{$t=-1.13$} & \multirow[b]{2}{*}{0.26} \\
\hline & & 57.46 & 25.56 & 76.70 & & \\
\hline \multirow{2}{*}{$\begin{array}{l}\text { Years of methamphetamine depen- } \\
\text { dency }\end{array}$} & & 5 & 4.7 & 5.06 & \multirow{2}{*}{$\mathrm{t}=-0.38$} & \multirow{2}{*}{0.70} \\
\hline & & 2.58 & 2.58 & 2.65 & & \\
\hline Cigarette smoker participants & & $28(93.33)$ & $13(86.6)$ & $15(100)$ & $X^{2}=2.14$ & 0.14 \\
\hline $\begin{array}{l}\text { Participants with an opium abuse his- } \\
\text { tory }\end{array}$ & & $8(26.7)$ & $5(33.3)$ & $3(20)$ & $X^{2}=0.68$ & 0.40 \\
\hline Participants with a heroin abuse history & & $7(23.3)$ & $3(20)$ & $4(26.7)$ & $x^{2}=0.18$ & 0.66 \\
\hline $\begin{array}{l}\text { Participants with a Crack-heroin abuse } \\
\text { history }\end{array}$ & & $3(10)$ & $3(20)$ & $0(0)$ & $X^{2}=3.33$ & 0.06 \\
\hline $\begin{array}{l}\text { Participants with a cannabis abuse } \\
\text { history }\end{array}$ & $\mathrm{n}(\%)$ & $10(33.3)$ & $5(33.3)$ & $5(33.3)$ & $X^{2}=0$ & 1.00 \\
\hline $\begin{array}{l}\text { Participants with an alcohol abuse } \\
\text { history }\end{array}$ & & $4(13.3)$ & $2(13.3)$ & $2(13.3)$ & $X^{2}=0$ & 1.00 \\
\hline Participants with a cocaine abuse history & & $1(3.3)$ & $0(0)$ & $1(6.7)$ & $X^{2}=1.03$ & 0.30 \\
\hline $\begin{array}{l}\text { Participants with a tramadol abuse } \\
\text { history }\end{array}$ & & $7(23.3)$ & $4(26.7)$ & $3(20)$ & $X^{2}=7.6$ & 0.66 \\
\hline
\end{tabular}

Ilranian Rehabilitation Journa

Effect of repeated tDCS on cue-induced craving:

According to Levene's test, the assumption of the equality of variances of the two groups is met $(\mathrm{F}=0.14$, $\mathrm{P}>0.05)$. The ANCOVA also indicates that the active tDCS has significantly decreased induced craving $(\mathrm{F}=7.91, \mathrm{P}<0.05)$ (Table 3$)$.

Effect of repeated tDCS on instant craving: because the assumptions for ANCOVA were not met, the differential t-test was used to analyze the results. There was no significant difference between active tDCS and sham tDCS in terms of reducing instant craving $(\mathrm{F}=2.24, \mathrm{P}>0.05)$ (Table 4).

\section{Discussion}

The present study aimed at examining the effect of repeated sessions of bilateral tDCS over DLPFC, us- 
Table 2. Positive and negative affect

\begin{tabular}{|c|c|c|c|c|c|}
\hline- & - & Mean $\pm S D$ & $\mathbf{t}$ & df & P-value \\
\hline \multirow{2}{*}{ Pretest } & Positive affect & $35.60 \pm 8.78$ & -1.08 & 28 & 0.28 \\
\hline & Negative affect & $32.56 \pm 8.71$ & 1.29 & 28 & 0.20 \\
\hline \multirow{2}{*}{ Posttest } & Positive affect & $36.26 \pm 8.67$ & -0.79 & 28 & 0.43 \\
\hline & Negative affect & $27.20 \pm 9.03$ & 0.60 & 28 & 0.55 \\
\hline
\end{tabular}

Table 3. One-way ANCOVA analyzing the effectiveness of tDCS on drug craving in patients with a methamphetamine use disorder

\begin{tabular}{ccccccc}
\hline Source & Type III Sum of Squares & df & Mean Square & $\mathbf{F}$ & Sig. & Partial Eta Squared \\
\hline Intercept & 0.006 & 1 & 0.006 & 0.000 & 0.997 & 0.001 \\
\hline Pretest & 5329.954 & 1 & 5329.954 & 10.710 & 0.003 & 0.284 \\
Study groups & 3936.211 & 1 & 3936.211 & 7.910 & 0.009 & 0.227 \\
Error & 13436.713 & 27 & 497.656 & - & - & - \\
Total & 66975.000 & 30 & - & - & - & - \\
\hline
\end{tabular}

Iranian Rehabilitation Đournal

ing anodal stimulation of right hemisphere and cathodal stimulation of the left hemisphere on methamphetamine craving for the rehabilitation of abstinent-methamphetamine users. The results indicated that repeated bilateral tDCS over DLPFC was not effective in reducing selfreport instant craving, but reduced self-reported craving in the craving induction condition. In fact, even though tDCS had no significant effect on the subjective feeling of craving, it reduced methamphetamine craving in the craving induction condition. The results of the study indicate that craving induction to assess craving changes in the short time provides a more accurate index of craving because it faces the subject with a situation close to the actual situation; but, craving assessment using the DDQ relies more on the person's subjective feelings. In another clinical trial aimed at exploring the effect of tDCS on methamphetamine craving, also different results were found for the craving with and without induction [21] The long-term or different forms of intervention may be needed to change the subjective feeling of craving.

Although non-invasive brain stimulation methods, including transcranial electrical stimulation and transcranial magnetic stimulation, have been extensively studied as new approaches to reduce craving and to rehabilitate patients with substance-related disorders [18], only one previous clinical trial has focused on the effectiveness of tDCS in treating methamphetamine use disorder; in this clinical trial, one session of one-sided anodal stimulation of right DLPFC immediately reduced drug craving in the rest condition, but increased craving when the participants were shown methamphetamine-related pictures [21]. The authors supposed that the increase in craving observed in the induction

Table 4. Results of differential t-test for analyzing the effect of tDCS on drug craving in patients with a methamphetamine use disorder

\begin{tabular}{|c|c|c|c|c|c|c|c|c|c|}
\hline \multirow{2}{*}{ - } & \multirow{2}{*}{-} & \multirow{2}{*}{ Mean $\pm S D$} & \multirow{2}{*}{$\mathbf{N}$} & \multicolumn{3}{|c|}{ Levene's Test } & \multicolumn{3}{|c|}{ t-test } \\
\hline & & & & Variances & $\mathbf{F}$ & $\mathbf{P}$ & $\mathbf{t}$ & df & $\mathbf{P}$ \\
\hline \multirow{2}{*}{ DDQ } & Active & $-13.266 \pm 12.89$ & 15 & Equal & 2.24 & 0.146 & -0.332 & 28 & 0.743 \\
\hline & Sham & $-11.60 \pm 14.57$ & 15 & Not equal & - & - & -0.332 & 27.58 & 0.743 \\
\hline
\end{tabular}


condition may have been owing to sustained attention and, in fact, better cognitive processing of craving-related pictures [21]. These results are not consistent with the results of the study. This discrepancy might result from the difference in the number of sessions or different montages used by the two studies.

In the previous studies, one session of tDCS with the same electrodes assortment as the one applied in the present study (anodal stimulation of the right DLPFC and cathodal stimulation of the left DLPFC) resulted in the reduction of craving for alcohol, nicotine, marijuana, and food [17]. In addition, 5 sessions with this montage have led to an increase in abstinence time in 3-month follow-up and suppression of craving for at least one week in crack-cocaine users $[18,25]$. In another study, 5 sessions of bilateral tDCS (right anodal/left cathodal) reduced cigarette craving [19]. These findings were in agreement with those of the present study in terms of induced craving reduction.

We targeted DLPFC; therefore, the observed changes must have happened because of a change in this region. In fact, other studies targeting DLPFC have shown that the modulation of this region is related to improvement in the cognitive performance in clinical and general populations and also mood changes in depressed patients [27-29]. Clinical trials focused on the effects of noninvasive techniques of brain stimulation on drug craving indicate that in terms of craving reduction, there is no significant difference between targeting the right or left DLPFC, using the non-invasive brain stimulation; however, more craving suppression has been reported when targeting the right DLPFC [15]. TDCS over the DLPFC can modify decision-making processes that have similar mechanisms to those of impulsivity in addiction [30]. Dunlop et al. believe that the positive effect of non-invasive brain stimulation techniques on drug craving results from improvement in the cognitive processes and reduction in the attentional bias toward drug-related cues [17].

Because of the ethical considerations, it was not possible in the present study to utilize craving induction methods, using drug-related pictures. Craving induction using drug-related pictures in residential rehab centers involves less ethical restrictions because drug use is not possible in these settings. Considering the difference between craving assessment methods that was found in the present study, the future studies are suggested to use more precise tools to induce craving, because if such a setting is used, the craving induction method using pictures can be employed; the attentional bias toward pictures can be assessed, using more precise methods, including Evoked
Response Potential. One of the limitations of this study was that the follow-up procedure was not successful. Future studies are suggested to have follow-up periods so that the effect of the intervention on abstinence duration can be assessed, and it can be determined whether the effect of the intervention on craving reduction has been maintained.

\section{Conclusion}

In this clinical trial, 5 sessions of bilateral tDCS over DLPFC with the anodal stimulation of right hemisphere and cathodal stimulation of left hemisphere reduced cueinduced craving in abstinent-methamphetamine users but had no significant effect on the subjective feeling of craving in these patients.

\section{Ethical Considerations}

\section{Compliance with ethical guidelines}

The present study was approved by the Ethics Committee of the University of Social Welfare and Rehabilitation Sciences (IR.USWR.REC.1395.283).

\section{Funding}

This study was derived from the MSc thesis of Mahsa Rohani Anaraki in Clinical Psychology at the University of Social Welfare and Rehabilitation Sciences, Tehran.

\section{Authors' contributions}

Conceptualization: Masoud Nosratabadi, Mahsa Rohani Anaraki, Behrouz Dolatshahi; Methodology: Behrouz Dolatshahi, Masoud Nosratabadi, Mahsa Rouhani Anaraki, Mohsen Nouri Yalghouzabadi; Investigation: Mahsa Rouhani Anaraki; Writing-original draft: Mahsa Rohani Anaraki, Sajede Rezaei; Writing-review, and editing: all of the authors; Funding acquisition: Mahsa Rohani Anaraki, Masoud Nosratabadi; Supervision: Behrouz Dolatshahi, Masoud Nosratabadi

\section{Conflict of interest}

The authors declare no Conflict of intere.

\section{Acknowledgments}

We hereby express our gratitude to Dooste-Khoob and Ghoghnous Center and its officials, as well as those clients who helped the researchers in this study. 


\section{References}

[1] United Nations Office on Drugs and Crime. World Drug Report 2017. Vienna : United Nations Office on Drugs and Crime (UNODC); 2017.

[2] Reichard EE, Nanaware-Kharade N, Gonzalez G, Thakkar S, Owens SM, Peterson EC. PEGylation of a High-Affinity Anti-(+) Methamphetamine Single Chain Antibody Fragment Extends Functional Half-Life by Reducing Clearance. Pharmaceutical Research. 2016; 33(12):2954-66. [DOI:10.1007/ s11095-016-2017-y] [PMID] [PMCID]

[3] Courtney KE. The effects of naltrexone on neural responses to methamphetamine cues: University of California, Los Angeles; 2016. [DOI:10.1016/j.drugalcdep.2016.08.141]

[4] Keoleian V, Polcin D, Galloway GP. Text messaging for addiction: A review. Journal of Psychoactive Drugs. 2015; 47(2):158-76. [DOI:10.1080/02791072.2015.1009200] [PMID] [PMCID]

[5] Wang G, Shi J, Chen N, Xu L, Li J, Li P, et al. Effects of length of abstinence on decision-making and craving in methamphetamine abusers. PlOS One. 2013; 8(7):e68791. [DOI:10.1371/journal.pone.0068791] [PMID] [PMCID]

[6] Pickens CL, Airavaara M, Theberge F, Fanous S, Hope BT, Shaham Y. Neurobiology of the incubation of drug craving. Trends in Neurosciences. 2011; 34(8):411-20. [DOI:10.1016/j. tins.2011.06.001] [PMID] [PMCID]

[7] American Psychiatric Association (APA). Diagnostic and statistical manual of mental disorders (DSM-5®). Washington, DC: American Psychiatric Association (APA); 2013.

[8] Su H, Zhong N, Gan H, Wang J, Han H, Chen T, et al. High frequency repetitive transcranial magnetic stimulation of the left dorsolateral prefrontal cortex for methamphetamine use disorders: A randomised clinical trial. Drug and Alcohol Dependence. 2017; 175:84-91. [DOI:10.1016/j.drugalcdep.2017.01.037] [PMID]

[9] Li X, Zeric T, Kambhampati S, Bossert JM, Shaham Y. The central amygdala nucleus is critical for incubation of methamphetamine craving. Neuropsychopharmacology. 2015; 40(5):1297-306. [DOI:10.1038/npp.2014.320] [PMID] [PMCID]

[10] Caprioli D, Venniro M, Zhang M, Bossert JM, Warren BL, Hope BT, et al. Role of dorsomedial striatum neuronal ensembles in incubation of methamphetamine craving after voluntary abstinence. Journal of Neuroscience. 2017; 37(4):1014-27. [DOI:10.1523/JNEUROSCI.3091-16.2016] [PMID] [PMCID]

[11] Narayanan NS, Laubach M. Inhibitory Control: Mapping Medial Frontal Cortex. Current Biology. 2017; 27(4):R148-R50. [DOI:10.1016/j.cub.2017.01.010] [PMID]

[12] Koob GF, Volkow ND. Neurocircuitry of addiction. Neuropsychopharmacology. 2010; 35(1):217-38. [DOI:10.1038/ npp.2009.110] [PMID] [PMCID]

[13] Ersche KD, Jones PS, Williams GB, Turton AJ, Robbins TW, Bullmore ET. Abnormal brain structure implicated in stimulant drug addiction. Science. 2012; 335(6068):601-4. [DOI:10.1126/science.1214463] [PMID]

[14] Dunlop K, Hanlon CA, Downar J. Noninvasive brain stimulation treatments for addiction and major depression. Annals of the New York Academy of Sciences. 2017; 1394(1):3154. [DOI:10.1111/nyas.12985] [PMID] [PMCID]
[15] Hone-Blanchet A, Ciraulo DA, Pascual-Leone A, Fecteau S. Noninvasive brain stimulation to suppress craving in substance use disorders: Review of human evidence and methodological considerations for future work. Neuroscience \& Biobehavioral Reviews. 2015; 59:184-200. [DOI:10.1016/j.neubiorev.2015.10.001] [PMID] [PMCID]

[16] Nitsche MA, Liebetanz D, Antal A, Lang N, Tergau F, Paulus W. Modulation of cortical excitability by weak direct current stimulation-technical, safety and functional aspects. Supplements to Clinical Neurophysiology. 2003; 56:255-76. [DOI:10.1016/S1567-424X(09)70230-2]

[17] Lefaucheur JP. A comprehensive database of published tDCS clinical trials (2005-2016). Neurophysiol Clinical. 2016; 46(6):319-98. [DOI:10.1016/j.neucli.2016.10.002] [PMID]

[18] Batista EK, Klauss J, Fregni F, Nitsche MA, NakamuraPalacios EM. A randomized placebo-controlled trial of targeted prefrontal cortex modulation with bilateral tDCS in patients with crack-cocaine dependence. International Journal of Neuropsychopharmacology. 2015; 18(12):1-11. [DOI:10.1093/ ijnp/pyv066] [PMID] [PMCID]

[19] Fecteau S, Agosta S, Hone-Blanchet A, Fregni F, Boggio $\mathrm{P}$, Ciraulo D, et al. Modulation of smoking and decisionmaking behaviors with transcranial direct current stimulation in tobacco smokers: Apreliminary study. Drug and Alcohol Dependence. 2014; 140:78-84. [DOI:10.1016/j.drugalcdep.2014.03.036] [PMID] [PMCID]

[20] den Uyl TE, Gladwin TE, Wiers RW. Transcranial direct current stimulation, implicit alcohol associations and craving. Biological Psychology. 2015; 105:37-42. [DOI:10.1016/j. biopsycho.2014.12.004] [PMID]

[21] Shahbabaie A, Golesorkhi M, Zamanian B, Ebrahimpoor M, Keshvari F, Nejati V, et al. State dependent effect of transcranial Direct Current Stimulation (tDCS) on methamphetamine craving. International Journal of Neuropsychopharmacology. 2014; 17(10):1591-8. [DOI:10.1017/S1461145714000686] [PMID]

[22] Shariatirad S, Vaziri A, Hassani-Abharian P, Sharifi Fardshad M, Molavi N, Fitzgerald PB. Cumulative and booster effects of tdcs sessions on drug cravings, lapse, and cognitive impairment in methamphetamine use disorder: A case study report. The American Journal on Addictions. 2016; 25(4):2646. [DOI:10.1111/ajad.12373] [PMID]

[23] Boggio PS, Liguori P, Sultani N, Rezende L, Fecteau S, Fregni F. Cumulative priming effects of cortical stimulation on smoking cue-induced craving. Neuroscience Letters. 2009; 463(1):82-6. [DOI:10.1016/j.neulet.2009.07.041] [PMID]

[24] Franken IH, Hendriks VM, van den Brink W. Initial validation of two opiate craving questionnaires: The Obsessive Compulsive Drug Use Scale and the Desires for Drug Questionnaire. Addictive Behaviors. 2002; 27(5):675-85. [DOI:10.1016/S0306-4603(01)00201-5]

[25] Sinha R, Fox HC, Hong K-iA, Hansen J, Tuit K, Kreek MJ. Effects of adrenal sensitivity, stress-and cue-induced craving, and anxiety on subsequent alcohol relapse and treatment outcomes. Archives of General Psychiatry. 2011; 68(9):942-52. [DOI:10.1001/archgenpsychiatry.2011.49] [PMID] [PMCID]

[26] Watson D, Clark LA, Tellegen A. Development and validation of brief measures of positive and negative affect: The PANAS scales. Journal of Personality and Social Psychol- 
ogy. 1988; 54(6):1063-70. [DOI:10.1037/0022-3514.54.6.1063] [PMID]

[27] Smith RC, Boules S, Mattiuz S, Youssef M, Tobe RH, Sershen $\mathrm{H}$, et al. Effects of transcranial Direct Current Stimulation (tDCS) on cognition, symptoms, and smoking in schizophrenia: A randomized controlled study. Schizophrenia Research. 2015; 168(1):260-6. [DOI:10.1016/j.schres.2015.06.011] [PMID]

[28] Dedoncker J, Brunoni AR, Baeken C, Vanderhasselt M-A. A systematic review and meta-analysis of the effects of transcranial Direct Current Stimulation (tDCS) over the dorsolateral prefrontal cortex in healthy and neuropsychiatric samples: Influence of stimulation parameters. Brain Stimulation. 2016; 9(4):501-17. [DOI:10.1016/j.brs.2016.04.006] [PMID]

[29] Meron D, Hedger N, Garner M, Baldwin DS. Transcranial Direct Current Stimulation (tDCS) in the treatment of depression: Systematic review and meta-analysis of efficacy and tolerability. Neuroscience \& Biobehavioral Reviews. 2015; 57:4662. [DOI:10.1016/j.neubiorev.2015.07.012] [PMID]

[30] Boggio PS, Zaghi S, Villani AB, Fecteau S, Pascual-Leone A, Fregni F. Modulation of risk-taking in marijuana users by transcranial Direct Current Stimulation (tDCS) of the Dorsolateral Prefrontal Cortex (DLPFC). Drug and alcohol dependence. 2010; 112(3):220-5. [DOI:10.1016/j.drugalcdep.2010.06.019] [PMID] 
This Page Intentionally Left Blank 